\title{
Improved Wind Speed Prediction Using Empirical Mode Decomposition
}

\author{
Yagang ZHANG ${ }^{1,2}$, Chenhong ZHANG ${ }^{1}$, Jingbin SUN ${ }^{1}$, Jingjing GUO ${ }^{1}$ \\ ${ }^{1}$ State Key Laboratory of Alternate Electrical Power System with Renewable Energy Sources, North \\ China Electric Power University, Beijing, 102206, China \\ ${ }^{2}$ Interdisciplinary Mathematics Institute, University of South Carolina, \\ Columbia, SC 29208, United States \\ yagangzhang@ncepu.edu.cn
}

\begin{abstract}
Wind power industry plays an important role in promoting the development of low-carbon economic and energy transformation in the world. However, the randomness and volatility of wind speed series restrict the healthy development of the wind power industry. Accurate wind speed prediction is the key to realize the stability of wind power integration and to guarantee the safe operation of the power system. In this paper, combined with the Empirical Mode Decomposition (EMD), the Radial Basis Function Neural Network (RBF) and the Least Square Support Vector Machine (SVM), an improved wind speed prediction model based on Empirical Mode Decomposition (EMD-RBF-LS-SVM) is proposed. The prediction result indicates that compared with the traditional prediction model (RBF, LS-SVM), the EMDRBF-LS-SVM model can weaken the random fluctuation to a certain extent and improve the short-term accuracy of wind speed prediction significantly. In a word, this research will significantly reduce the impact of wind power instability on the power grid, ensure the power grid supply and demand balance, reduce the operating costs in the grid-connected systems, and enhance the market competitiveness of the wind power.
\end{abstract}

Index Terms-renewable energy, wind speed prediction, empirical mode decomposition (EMD), radial basis function neural network (RBF), least squares support vector basis (LSSVM).

\section{INTRODUCTION}

The sharp decrease of traditional energy and the aggravation of environmental pollution have a negative impact on the survival and development of human beings. Laszlo VARRO, the chief economist of the International Energy Agency (IEA) [1], pointed out that the total global energy investment in 2016 was about 17 thousand dollars, of which the share of the low-carbon industry increased by twelve percentage points over the same period, reaching to $43 \%$, while that of oil and natural gas accounted for $40 \%$, the low carbon industry surpassed the fossil fuel supply and became the largest industry in energy investment industry for the first time. The rapid development of the green energy has been coping the global energy crisis and environmental deterioration, thus promoting a virtuous circle of energy and environment.

Wind power is considered as one of the fastest growing sources of energy in the world recently due to its relatively mature technologies, higher cost efficiency and resource

This research was supported partly by the National Natural Science Foundation of China (51637005), the Fundamental Research Funds for the Central Universities (2017MS166) and the Natural Science Foundation of Hebei Province (G2016502009). effectiveness. According to the statistics of Global Wind Energy Council [2], the global installed capacity of wind power has increased from $23.9 \mathrm{GW}$ to $486.7 \mathrm{GW}$ from December 31, 2001 to December 31, 2016, and the compound annual growth rate is $22.25 \%$, with a cumulative increase of $12.5 \%$ over the same period. However, due to the uneven distribution of traditional energy and renewable energy and insufficient power grid capacity, all countries have different degrees of abandonment. Reliable wind power prediction facilitates the power dispatching department to adjust the dispatching plan in time, formulates the wind farm control strategies, and promotes wind power to be configurable; at the same time, it mitigates the impact of wind power instability on the power grid and significantly reduces the power grid rotating reserve capacity, thereby reducing the power generation cost and maintain market competitiveness [3].Therefore, the improved wind speed and power forecasting methods for the wind farms has become the research focus on the wind power development. Among them, the wind speed prediction is the basic work and a key link.

In general, the wind speed prediction methods are divided into four categories, including physical methods, statistical methods, artificial intelligence techniques and combined forecasting methods. The physical method which is combining with the geographical factors is the process of analysis and calculation on the basis of improving the resolution of the numerical weather forecast (NWP). Statistical method is establishing a mapping relationship between input and output based on a large number of historical data, and the commonly used methods include grey prediction [4-6], time series prediction model [7-9] and so on. The artificial intelligence technology describes the nonlinear relationship between system's inputs and outputs by means of artificial intelligence. The Artificial neural network [10-13], the support vector machine (SVM) [14, $15]$ and so on are commonly used. In order to improve the forecasting accuracy, researchers around the world have tried to combine different forecasting methods and have obtained many ideal forecasting results. Paper [16] proposes a networked linear control model using a multi-objective genetic algorithm that is optimized, and the model achieves a simpler and more effective networked control. Paper [17] proposed a fuzzy control method by studying the stability conditions of the controlled equipment. This method is applicable to the control of a class of arbitrary nonlinear power stations. The essence of the above several kinds of 
prediction methods is directly matches the wind speed sequence and the influencing factors, and its prediction effect is overly dependent on the model prediction performance. In reality, the wind speed sequence has the strong non-stationary and non-linear characteristics as well as the periodic variation with different nested cycles [18]. Therefore, it can be regarded as the superposition of several different frequency components. Empirical Mode Decomposition (EMD) method is used to decompose the original wind speed data into an intrinsic mode function (IMF) with local time-varying features adaptively, and it can reduce its nonstationarity effectively. By using a large number of actual data to simulate, we finds that the prediction error mainly comes from high frequency components. Based on this, a combined wind speed prediction model based on empirical mode decomposition is established in this paper, namely, then we separate the high frequency component and the low frequency component based on the mean instantaneous frequency, next we use the least squares support vector machine (LS-SVM) to establish the prediction model of high frequency components, and use the RBF neural network (RBF) to establish the prediction model of low frequency component and the remainder, finally, the prediction results of each component are added to get the final wind speed prediction sequence. Compared to the traditional prediction methods, this method digs out the characteristics of the wind speed series deeply, reduces the random fluctuation of the wind speed sequence to a certain extent and effectively improves the prediction accuracy. The structure of this paper is as follows: The sections 2 introduces the Empirical Mode Decomposition method and the numerical algorithm. The section 3 describes the modeling process of EMD-RBF-LSSVM model. The section 4 presents the comparison of prediction results and error analysis of each model. The summary and prospect of the full text are in the section 5 .

\section{EMPIRICAL Mode DeCOMPOSITION AND THE NUMERICAL ALGORITHM}

Empirical Mode Decomposition (EMD) is an adaptive screening method that can screen the trend of different features existing in complex signals step by step to obtain several intrinsic mode functions (IMF) from high frequency to low frequency and the intrinsic mode functions need to meet the following two conditions [19-21]:

(1) In the whole data interval, the number of the extreme points is equal to the number of zero-crossing points or at most one difference;

(2) At any point, the mean value of the envelope defined by the local maximum and minimum point are zero.

For a given original wind speed sequence $\{x(t)\}$, the steps of empirical mode decomposition are as follows [22]:

Step 1 Find the position and amplitude of all the local maximum and minimum values in the signal;

Step 2 The spline interpolation of the local maximum value composes the upper envelope, and the spline interpolation of the local minimum value composes the enveloping lower line, represented by $e_{\max }(t)$ and $e_{\min }(t)$ respectively;
Step 3 In each time period t, the average of upper and lower envelope is calculated as

$$
m_{1}=\frac{e_{\max }(t)+e_{\min }(t)}{2}
$$

Step 4 Minus the average envelope of the input signal: $h_{1}(t)=x(t)-m_{1}(t)$. This is an iteration of the screening process. The next step is to check if $h_{1}(t)$ is an intrinsic model function. In Huang's original work, when the difference between two successive screens was less than a selected threshold SD, the screening process stopped and SD was defined as

$$
S D=\sum_{t=0}^{T}\left[\frac{\left|h_{1(k-1)}(t)-h_{1 k}(t)\right|^{2}}{h_{1(k-1)}^{2}(t)}\right]
$$

In this case, $h_{11}=h_{1}-m_{11}$ if this is the kth iteration, then $h_{1 k}=h_{1(k-1)}-m_{1 k}$.

Step 5 When all the conditions are satisfied, the intrinsic mode function is defined as $C_{1}=h_{1 k}$, after deriving the intrinsic mode function $c_{1}=h_{1 k}$, we define the remainder as $r_{1}(t)=x(t)-c_{1}(t)$.

Step 6 Take the remaining amount as the input signal, then repeat the step $1-6$. Therefore, the result is

$$
r_{1}-c_{1}=r_{2}, \cdots, r_{n-1}-c_{n}=r_{n}
$$

The empirical mode decomposition process is completed when the residual value does not contain any extreme points. This means that the residual value can be constant or monotone functions. The signal can be expressed as the sum of the intrinsic mode functions and the final residual values:

$$
x(t)=\sum_{i=1}^{n} c_{i}(t)+r_{n}
$$

The inherent modal functions extracted are symmetric and have a unique local frequency, and at the same time, the different intrinsic modal functions do not show the same frequency.

For each of the IMF's Hilbert transformations:

$$
y_{j}=\frac{1}{\pi} P \int_{-\infty}^{+\infty} \frac{C_{i}(\tau)}{t-\tau} d \tau
$$

In the formula, $\mathrm{P}$ is the Cauchy principal value, $C_{i}(\tau)$ is a signal.

The analytical signal $z_{j}(t)=c_{i}(t)+i y_{j}(t)$, is expressed as polar coordinates:

$$
z_{j}(t)=a_{i}(t) e^{i \theta_{j}}
$$

Where

$$
\begin{aligned}
& a_{i}(t)=\left[c_{j}^{2}(t)+y_{j}^{2}(t)\right]^{\frac{1}{2}} \\
& \theta_{j}=\arctan \left[\frac{y_{j}(t)}{c_{j}(t)}\right]
\end{aligned}
$$


The instantaneous frequency of each IMF is:

$$
\omega_{j}(t)=\frac{d}{d t} \theta_{j}(t)
$$

Radial Basis Function (RBF) is a kind of feed forward neural network structure proposed by J. Moody and C. Darken in the late 1980s. It is an artificial neural network that uses a locally accepted domain to perform function mapping based on biological local regulation and overlapping acceptance region knowledge [23].

$$
\varphi(x, c)=\varphi(x-c)
$$

Where $\varphi$ is the radial basis function, $c$ is the center and $\|\cdot\|$ is set as Euclidean distance in general. The most commonly used radial basis function is the Gaussian function:

$$
\varphi(r)=\exp \left(-r^{2} / \sigma^{2}\right)
$$

Where $r$ is the Euclidean distance from $X$ to $C$.

The objective function from the input layer to output layer is:

$$
y(x)=\sum_{i=1}^{k} \omega_{i} \varphi\left(\left\|x-c_{i}\right\|\right)
$$

Among them, $y(x)$ represents results of the output layer, $x$ is the output layer of the vector, $\mathrm{k}$ is the number of the hidden layer nodes, $\varphi\left(\left\|x-c_{i}\right\|\right)$ is the activation function of the $i$ hidden layer node, $c_{i}$ is the center of the $i$ hidden layer, $\omega_{i}$ is the connection of the $i$ hidden layer.

LS-SVM is an improvement to SVM [20]. It changes the inequality constraint of traditional SVM to equality constraint and takes the error square sum loss function as the empirical loss of training set, transforming the solution of quadratic programming problem into the solving linearity equations, which increases the speed of solving and the accuracy of convergence [24-26].

A sample can be expressed as $\left(x_{1}, y_{1}\right),\left(x_{2}, y_{2}\right), \cdots ;\left(x_{n}, y_{n}\right)$, firstly, a nonlinear mapping $\varphi(\cdot)$ is used to map the sample from the original space $R^{n}$ to the feature space $\varphi\left(x_{i}\right)$, and the optimal decision function is constructed in this high dimensional space [27]:

$$
y(x)=\omega \varphi(x)+b
$$

Where $\varphi(x)$ is the mapping function, $\omega$ is the weight vector and $\mathrm{b}$ is the constant.

Use the principle of structural risk minimization to find $\omega, b$. The objective function and restrictions are:

$$
\min J(\omega, \zeta)=\frac{1}{2} \omega^{T} \omega+\frac{1}{2} r \sum_{i=1}^{n} \zeta_{i}^{2}
$$$$
\text { s.t. } \quad y_{i}=\varphi\left(x_{i}\right)+b+\zeta_{i}
$$

Where $\zeta_{i}$ is the relaxation factor, $r$ is the penalty parameter. The Lagrange function is constructed as:

$$
L(\omega, b, \zeta, \alpha)=\frac{1}{2} \omega^{T} \omega+\frac{1}{2} r \sum_{i=1}^{n} \zeta_{i}^{2}-\sum_{i=1}^{n} \omega \varphi\left(x_{i}\right)+b
$$

Where, $\alpha_{i}$ is the Lagrange Multiplier.

By the conditions of KTT

$$
\left\{\begin{array}{c}
\frac{\partial L}{\partial \omega}=0 \Rightarrow \omega=\sum_{i=1}^{n} \alpha_{i} \varphi\left(x_{i}\right) \\
\frac{\partial L}{\partial b}=0 \Rightarrow \sum_{i=1}^{n} \alpha_{i}=0 \\
\frac{\partial L}{\partial \zeta}=0 \Rightarrow \alpha_{i}=r \zeta_{i} \\
\frac{\partial L}{\partial \alpha}=0 \Rightarrow \omega^{T} \varphi\left(x_{i}\right)+b+\zeta_{i}-y_{i}=0
\end{array}\right.
$$

The elimination of $\omega, \zeta$ in (14) is available:

$$
\left[\begin{array}{cc}
0 & 1^{T} \\
1 & K+r^{-1}
\end{array}\right]\left[\begin{array}{l}
b \\
a
\end{array}\right]=\left[\begin{array}{l}
0 \\
y
\end{array}\right]
$$

Where

$$
\begin{aligned}
& 1=[1,1, \cdots, 1], K=\left\{k_{i j}\right\}=\left\{\varphi\left(x_{i}\right)^{T} \varphi\left(x_{j}\right)\right\} \\
& y=\left[y_{1}, y_{2}, \cdots, y_{n}\right]
\end{aligned}
$$

The increase of dimension after space mapping leads to the complexity of calculation and the increase of computation. According to the functional theory, the inner product calculation of the high-dimensional space can be equivalent to a kernel function of the original input space, so the inner function $\varphi\left(x_{i}\right) \cdot \varphi\left(x_{j}\right)$ can be replaced by the kernel function $K\left(x_{i}, x_{j}\right)$, Thus the high dimensional problem is solved. The Least Square method is used to solve the above equation to get $a, b$, and the least squares support vector machine model is:

$$
y(x)=\sum_{i=1}^{n} \alpha_{i} K\left(x_{i}, x_{j}\right)+b
$$

In the formula, $K\left(x_{i}, x_{j}\right)$ is kernel function, $K\left(x_{i}, x_{j}\right)=\varphi\left(x_{i}\right) \cdot \varphi\left(x_{j}\right)$, which is equal to the inner product of $\varphi\left(x_{i}\right)$ and $\varphi\left(x_{j}\right)$ the eigenvector $x_{i}$ and $x_{j}$ in its characteristic space $\varphi\left(x_{i}\right)$ and $\varphi\left(x_{j}\right)$.

\section{The Combined Wind SPEed PREDiction Model BASED ON EMD (EMD-RBF-LS-SVM)}

The wind speed data used in the simulation experiments in this paper are all from the Sotavento wind farm in Galicia, Spain [28]. Galicia is located in northwest Spain, facing the Atlantic Ocean, rich in wind resources. There are four seasons in a year. Under the control of the subtropical high in summer, it is hot and dry and affected by the west wind in winter, it is mild and rainy. The kind of climate characteristics is unique in all kinds of climate in the world.

In general, the three months in a certain season tend to have similar weather conditions [29]. Thus, we separately 
choose one month from each season as their representative. Therefore, the wind speed data collected every 10 minutes in February, May, August and November, are used to verify the adaptability of the research method to wind speed in different seasons. Figure 1 shows the actual wind speed distribution over the past four months. The prediction model data usage as shown in Table I, the maximum wind speeds in May and August are only $16.27 \mathrm{~m} / \mathrm{s}$ and $14.41 \mathrm{~m} / \mathrm{s}$, respectively. The wind speed changes stably. The maximum wind speed in November is $20.83 \mathrm{~m} / \mathrm{s}$, which fluctuates more than in the previous two months. Affected by the westerly wind, the fluctuation in February reaches the maximum, the minimum wind speed is $0.35 \mathrm{~m} / \mathrm{s}$, the maximum wind speed is $38.93 \mathrm{~m} / \mathrm{s}$.

Using EMD to decompose the four-month original wind speed sequences. The decomposition results are shown in Figure 2. In each exploded picture, the first figure represents the original wind speed signal, the red curve in the last figure represents the remainder describing the trend of the wind speed sequence. There are eight IMF components separately in February, August and November, May have seven IMF components.

In each month, the IMF components are respectively subjected to Hilbert transform, and according to the instantaneous frequency formula, calculate the instantaneous frequency of each component, and then obtain the average value. Table II shows the four-month instantaneous frequency average value of each IMF component. From the average instantaneous frequency of each component in February and August, the average instantaneous frequency of IMF3 has a large gap with the mean of the average instantaneous frequency of IMF2, so IMF1 and IMF2 are determined as the high frequency components and IMF3IMF8 are the low frequency components. In May and November, the average instantaneous frequency of IMF4 is obviously less than the average instantaneous frequency of IMF3, so the high frequency component in May and November is IMF1-IMF3, the Low-frequency component in May is IMF4-IMF7 and the Low-frequency component in November is IMF4-IMF8.

The prediction method of this study is as follows:

Step 1 Use EMD method decompose the original wind speed sequence. The original wind speed sequence is decomposed into several IMF components and the remainder, which show the trend of wind speed sequence;

Step 2 The determination of the IMF prediction components at different frequencies. Each IMF components are subjected to Hilbert transform, we can obtain the instantaneous frequency, According to the feature that the highest frequency goes to the lowest one, calculate the instantaneous frequency of each component, then obtain the average sequence of each IMF. In this sequence, a distinction is made on the basis of a significant decrease in the high frequency components, the former is identified as the higher frequency component, and the latter is identified as the low frequency component.

Step 3 The prediction of high frequency component. The high-frequency components obtained by the decomposition of the original wind speed sequence, have higher frequency values and larger fluctuations in frequency, including highfrequency volatility and random components. Therefore, the
LS-SVM, which has strong fitting ability to complex nonlinear problems, is used to establish the forecasting model for each high frequency component, and obtain each prediction value and sum each prediction value up to obtain the final high frequency prediction value $\widehat{y}_{1}(t)$;

Step 4 The prediction of Low-frequency components and the remainder of. The RBF neural network is used to establish the forecasting models for the low-frequency components, and the predicted values are obtained and added together to obtain the final low frequency prediction value $\widehat{y}_{2}(t)$;

Step 5 The high-frequency and low-frequency prediction value is added to obtain the final result $\hat{y}(t)$, that is $\widehat{y}(t)=\widehat{y}_{1}(t)+\widehat{y}_{2}(t) ;$

Step 6 The analysis of the model error. In this paper, two error indexes, average absolute error (MAE) and root mean square error (RMSE), are used to quantitatively evaluate the forecasting results. The specific formulas are as follows:

$$
\begin{aligned}
M A E & =\frac{1}{k} \sum_{t=1}^{k}|y(t)-\hat{y}(t)| \\
R M S E & =\sqrt{\frac{1}{k} \sum_{t=1}^{k}(y(t)-\hat{y}(t))^{2}}
\end{aligned}
$$

Among them, $y(t)$ is the true wind speed value and $\widehat{y}(t)$ is the wind speed forecasting value.

Follow the detailed modeling steps above we can get the model flow chart, as shown in figure 3.

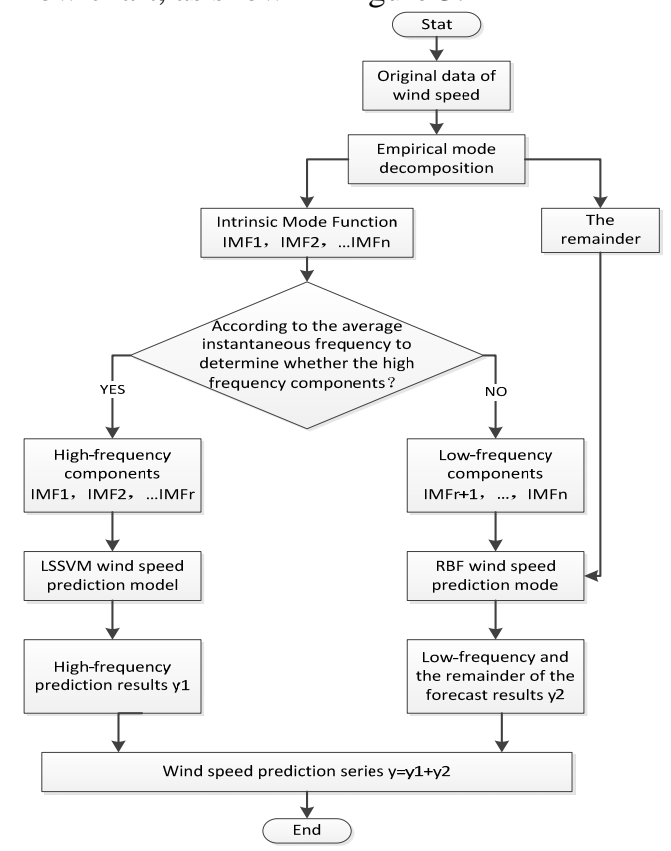

Figure 3. Modeling flow chart of EMD-RBF-LS-SVM wind speed forecasting model

TABLE I. STATISTICS OF WIND CONDITIONS IN FEBRUARY, MAY, AUGUST

\begin{tabular}{|c|c|c|c|c|}
\hline \multicolumn{5}{|c|}{ AND NOVEMBER } \\
\hline Data collection time & 2 & 5 & 8 & 11 \\
\hline Sample sizes & 1200 & 1500 & 1150 & 1350 \\
\hline Average wind velocity & 6.674 & 6.008 & 6.1994 & 9.2685 \\
\hline Maximum wind speed(m/s) & 38.93 & 16.27 & 14.41 & 20.83 \\
\hline Minimum wind speed(m/s) & 0.35 & 0.35 & 1.29 & 3.58 \\
\hline
\end{tabular}
AND NOVEMBER 


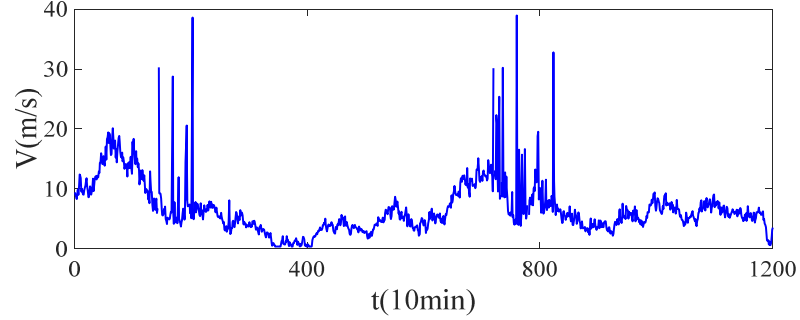

(a) Wind distribution in February

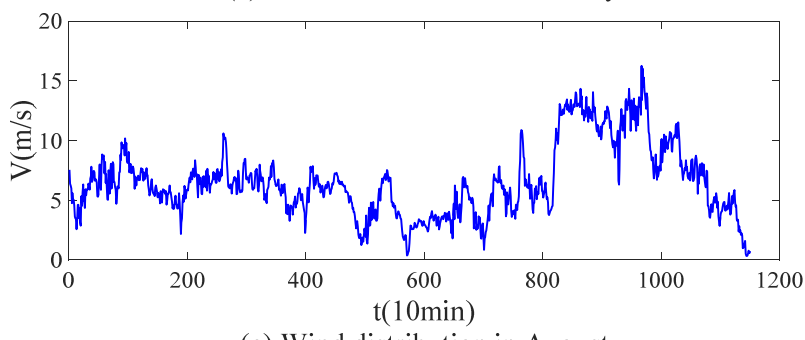

(c) Wind distribution in August

Figure 1. (a)-(d) Separately depict actual wind speed distribution in February, May, August and November in the Sotavento wind farm

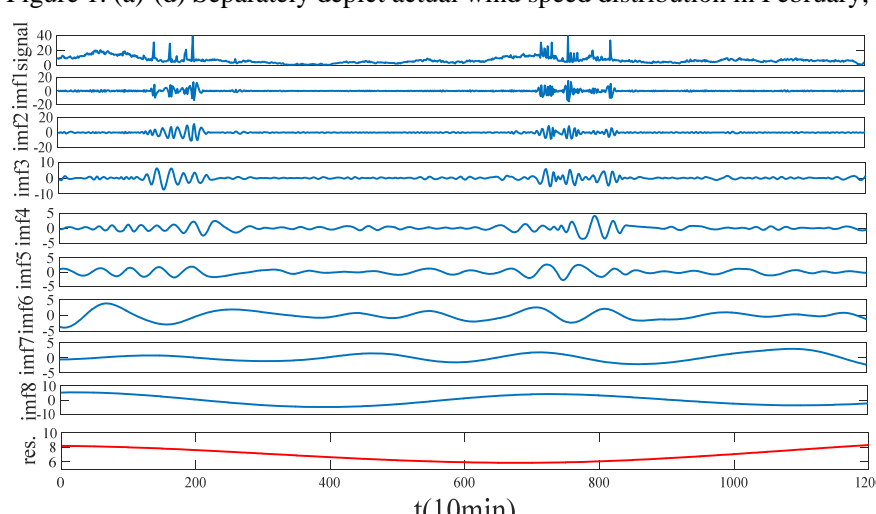

EMD decomposition results in February

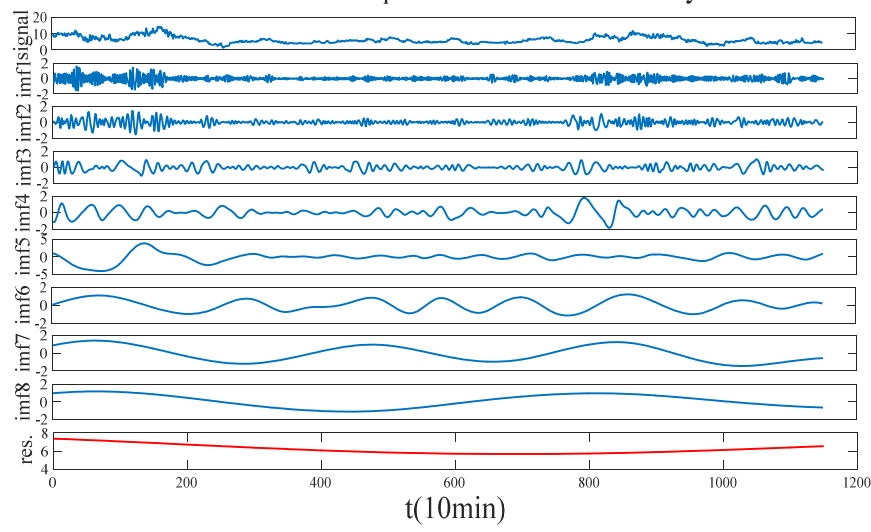

EMD decomposition results inAugust

Figure 2. EMD decomposition results in February, May, August and November

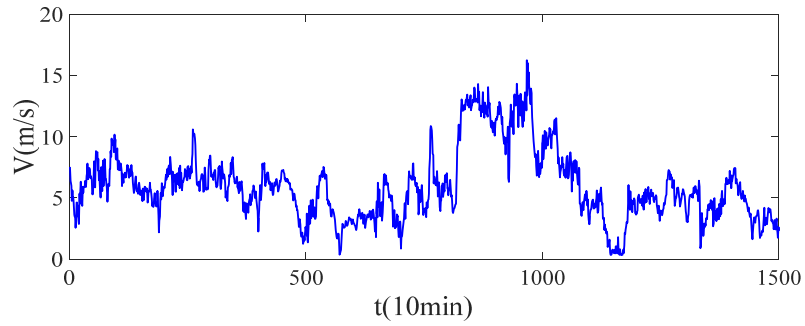

(b) Wind distribution in May

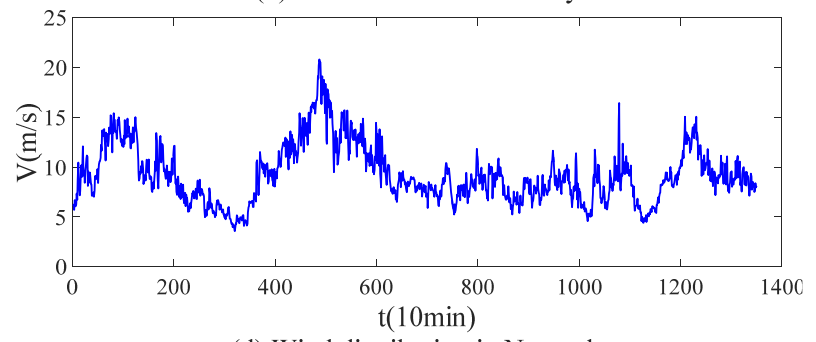

(d) Wind distribution in November

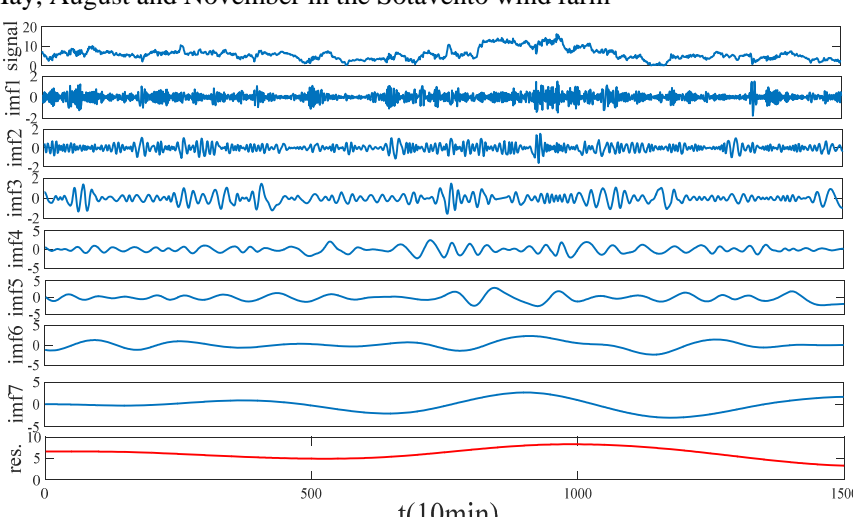

EMD decomposition results in May

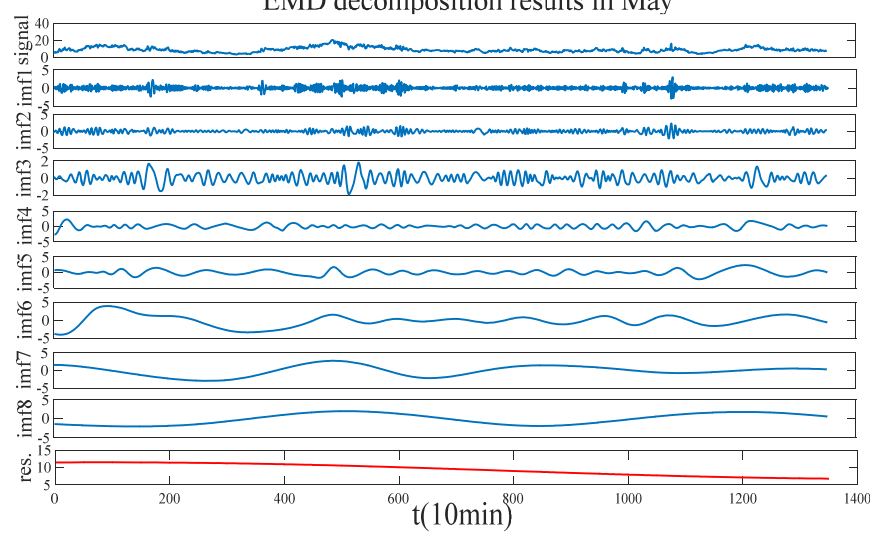

EMD decomposition results in November

TABLE II. INSTANTANEOUS FREQUENCY AVERAGE

\begin{tabular}{|c|c|c|c|c|c|c|c|c|}
\hline Component & IMF1 & IMF2 & IMF3 & IMF4 & IMF5 & IMF6 & IMF7 & IMF8 \\
\hline Month & & & & & & & & \\
\hline February & 0.1760 & 0.1946 & 0.0789 & 0.0654 & 0.0347 & 0.0147 & 0.0071 & 0.0028 \\
\hline May & 0.1704 & 0.1828 & 0.1481 & 0.0512 & 0.0232 & 0.0095 & 0.0040 & NUN \\
\hline August & 0.1713 & 0.1987 & 0.0821 & 0.0626 & 0.0276 & 0.0136 & 0.0049 & 0.0023 \\
\hline November & 0.1702 & 0.1935 & 0.1537 & 0.0579 & 0.0282 & 0.0108 & 0.0049 & 0.0027 \\
\hline
\end{tabular}

\section{EMD-RBF-LS-SVM MODEL RESULTS AND ERROR ANALYSIS}

According to the modeling process of the section III and the wind speed sequence modeling flow chart, we can get high frequency component prediction results and wind speed prediction results.

The stochastic volatility of the high frequency 
components brings many difficulties in improving the accuracy of wind speed prediction. The EMD method is used to decompose the wind speed sequence and extract the high frequency components. The least square support vector machine with strong non-linear fitting ability is used to model each high frequency component to obtain the prediction results and add them to obtain the high frequency component prediction result. The forecasting curves of the high frequency component in February, May, August and November are shown in Figure 4 (a) - (d) below. In the figure, the horizontal axis represents the prediction period, the vertical axis represents the high frequency component value, the blue line shows the actual high frequency component sequence, the red line represents the prediction result of the high frequency component, (a) and (d) represents the forecasting curve in February and November respectively, The prediction curves show that the highfrequency components fluctuate greatly, but the trend of their predicted curves is basically the same as the real ones, and they all fit the real ones. The first rapid decline at the thirteenth point in the figure (a) indicates that the LSSVM prediction model not only maintains a downward trend, but also does not differ significantly from the real data. Figure (b) and figure (c) are the predicted curves in May and November, respectively. The real high-frequency components are more stable and the prediction error is smaller. Thus, the separate prediction of the high-frequency components reduces the interference of the stochastic volatility to the prediction results, effectively improve the prediction accuracy of the high frequency component.

Based on the prediction algorithm in Section 3, the combined wind speed prediction model based on empirical mode decomposition is respectively established in different seasons. The RBF neural network and the least squares support vector machine LS-SVM, the traditional forecasting model, are used as the reference model.

Figures 5 (a) - (d) respectively describe the wind speed prediction curves in February, May, August and November. the reddish-brown band depict the wind speed prediction results of the RBF model, the red band depict that of the LSSVM model and the blue band depict that of the EMD-RBFLSSVM model. The black curves depict the actual wind speed distribution. The forecast results of different seasons show that both the RBF neural network prediction model and the LSSVM prediction model wind speed prediction model can describe the trend of the original wind speed series, but the values of the LSSVM prediction model are more close to the original wind speed data.The forecast results are better in May and August, because the wind speed fluctuate smaller. The predictions results of the two models are unsatisfactory and even worse in February. Figures (a) and (d) show the wind speed prediction in February and November. The wind sequence fluctuated greatly during the two-month forecast period. As shown in Figure (a), the first sharp decline trend occurs after the sixth point in the forecast period. We can see that the prediction curve of the RBF model only keeps the trend of the historical wind speed data and can not timely feed back the obvious change of the original wind speed sequence. Although the predicted curve of the LS-SVM model timely feedbacks its obvious change, its prediction error is very large. After each larger fluctuations, the wind speed forecasting results will have a greater deviation. On the contrary, the prediction results of the EMD-RBF-LSSVM model not only basically coincide with the actual wind speed series but also closer to the original wind speed series. This fully shows that the prediction results of the EMDRBF-LSSVM model are closer to the actual wind speed curve and can more accurately reflect the wind speed prediction result. Figures (b) and (c) show the wind speed prediction results in May and August, respectively, which shows that the EMD-RBF-LSSVM model also shows good performance when predicting a gentle wind speed sequence.
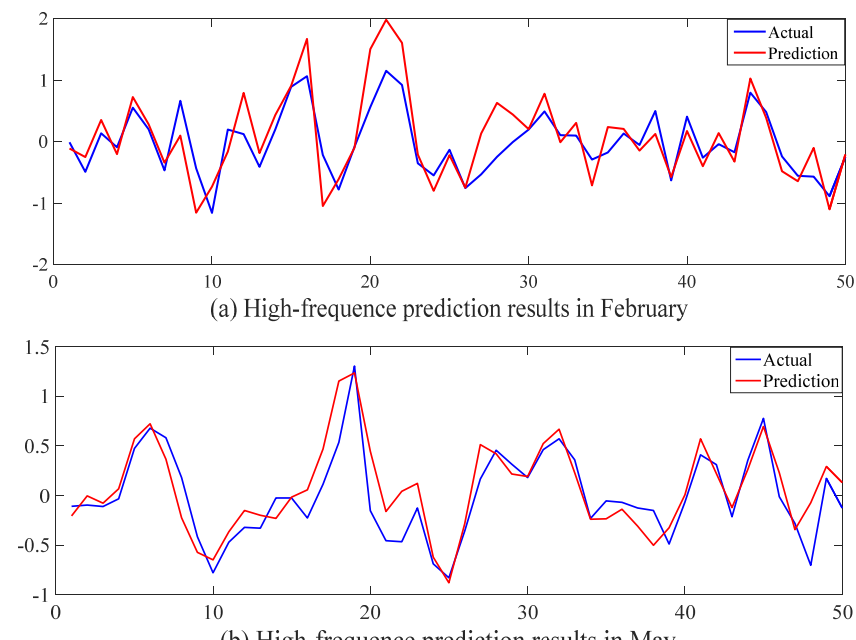

(b) High-frequence prediction results in May

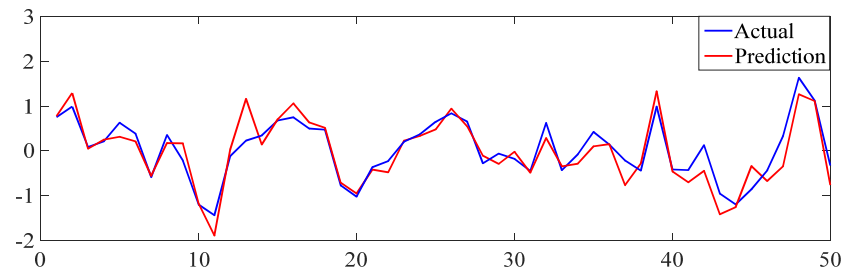

(c) High-frequence prediction results in August

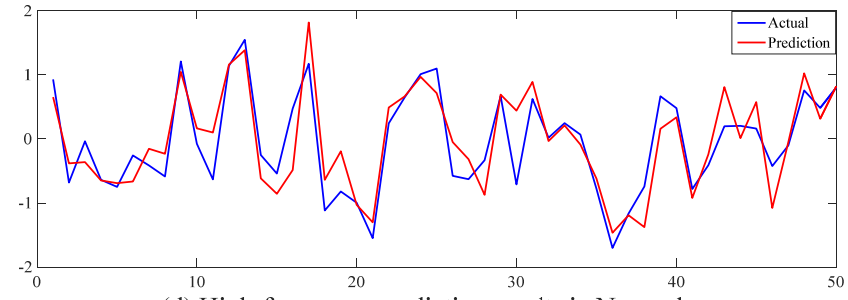

(d) High-frequence prediction results in November

Figure 4. (a)-(d) Separately depict the high frequency component prediction curves in February, May, August and November

The prediction results were quantitatively evaluated by using two error indexes, mean absolute error (MAE) and root mean square error (RMSE). Table III shows the prediction accuracy of the model. It can be seen that the EMD-RBF-LSSVM model shows the best prediction performance in November, compared with the LSSVM model, the MAE and RMSE values of the EMD-RBFLSSVM model decrease by at least $65 \%$, compared with the RBF model, that reduce at least $75 \%$, the same statistical data are at $63 \%$ and $68 \%$ in February, at $64 \%$ and $69 \%$ in May, and at $64 \%$ and $73 \%$ in August. The wind speed prediction accuracy of the EMD-RBF-LSSVM model is the highest in May, MAE and RMSE are 0.1768 and 0.2349, respectively. It can be seen that the wind speed prediction accuracy will be significantly improved when the wind 
speed sequence is stable. From the above analysis, for every wind speed sequence in different season, we know that the

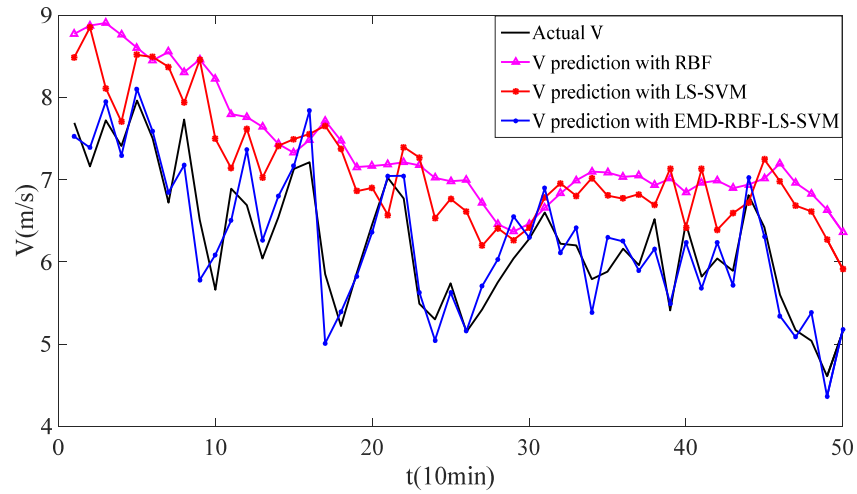

(a) Wind speed forecasting curve in February

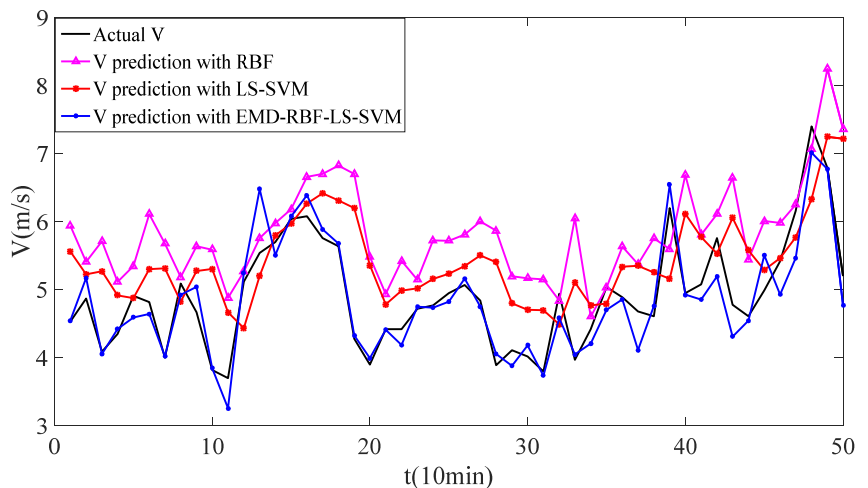

(c) Wind speed forecasting curve in August

Figure 5. The wind speed prediction curves in February, May, August and November error index of the EMD-RBF-LSSVM model is superior to the traditional wind speed prediction model.

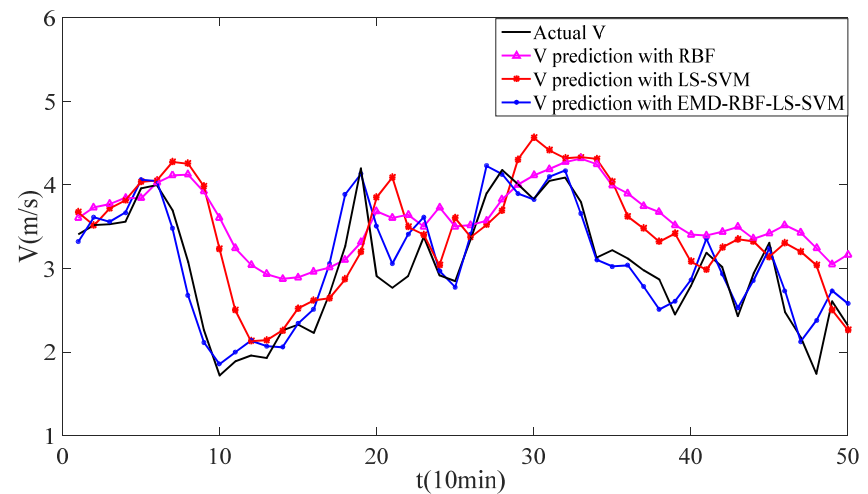

(b) Wind speed forecasting curve in May

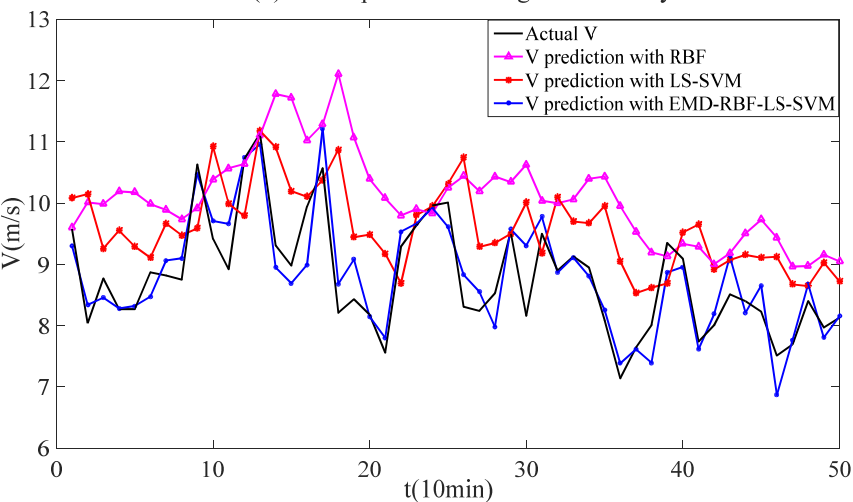

(d) Wind speed forecasting curve in November

TABLE III. The ERror Statistics Of POWER Prediction MOdeL

\begin{tabular}{|c|c|c|c|}
\hline \multirow{2}{*}{ Forecast cycle } & \multirow{2}{*}{ Prediction model } & \multicolumn{2}{|c|}{ Error indicators } \\
\cline { 2 - 4 } & RBF & 1.1013 & RMSE $(\mathrm{m} / \mathrm{s})$ \\
\hline \multirow{3}{*}{ February } & LSSVM & 0.9023 & 1.2591 \\
\cline { 2 - 4 } & EMD-RBF-LSSVM & 0.3024 & 1.0674 \\
\hline \multirow{3}{*}{ May } & RBF & 0.6390 & 0.3917 \\
\cline { 2 - 4 } & LSSVM & 0.5116 & 0.7767 \\
\cline { 2 - 4 } & EMD-RBF-LSSVM & 0.1768 & 0.6680 \\
\hline \multirow{5}{*}{ August } & RBF & 0.9420 & 0.2349 \\
\cline { 2 - 4 } & LSSVM & 0.6922 & 1.1236 \\
\cline { 2 - 4 } & EMD-RBF-LSSVM & 0.2210 & 0.8377 \\
\cline { 2 - 4 } & RBF & 1.3348 & 0.3005 \\
\hline \multirow{5}{*}{ November } & LSSVM & 0.9501 & 1.5889 \\
\cline { 2 - 4 } & EMD-RBF-LSSVM & 0.3019 & 1.1391 \\
\cline { 2 - 4 } & & & 0.3927 \\
\hline
\end{tabular}

\section{CONCLUSION}

As we all know, the over-consumption of conventional energy sources (coal, oil and natural gas) has led to energy shortages and environmental pollution. Reducing the burning of fossil fuels and accelerating the development and utilization of renewable energy have become the common consensus and concerted action for all countries in the world. So it is highly concerned by governments, institutions and enterprises that wind power is an effective way to mitigate climate change, improve energy security and promote low carbon growth.

How to improve the accuracy of wind speed prediction is a hot issue in wind power development. The traditional prediction methods can't meet the requirements of accuracy. Therefore, in this paper, a new wind speed forecasting model based on empirical mode decomposition is put forward. Using the empirical mode decomposition method to excavate the wind speed sequence characteristics and selecting the appropriate prediction method can eliminate the disturbance for the wind speed prediction, then effectively improve the wind power forecasting reliability, realize the accurate prediction for the wind farm power generation, and lay a solid foundation for vigorously promoting the wind power industry. 


\section{ACKNOWLEDGMENT}

The authors thank Distinguished Prof. Eugen COCA and the anonymous referees for the thoughtful and constructive suggestions that led to a considerable improvement of the paper.

\section{REFERENCES}

[1] International Energy Agency (IEA).World Energy Outlook 2017 [DB/OL]

[2] Global Wind Energy Council (GWEC). Global Statistics [DB/OL].

[3] J.Z. Wang, Y.L. Song, F. Liu, R. Hou. "Analysis and application of forecasting models in wind power integration: A review of multi-stepahead wind speed forecasting models," Renewable and Sustainable Energy Reviews, vol. 60, pp.960-981, Feb.2016.doi:10.1016/j.rser.2016.01.114

[4] R. Rajesh "Forecasting supply chain resilience performance using grey prediction,” Electronic Commerce Research and Applications, Vol.20, pp.42-58, sep.2016. doi:10.1016/j.elerap.2016.09.006

[5] Y.G. Zhang, Y. Xu, Z.P. Wang, "GM $(1,1)$ grey prediction of Lorenz chaotic system,” Chaos, Solitons and Fractals, vol. 42, pp. 1003-1009, Feb. 2009. doi: 10.1016/j.chaos.2009. 02.031

[6] A. Bezuglov, G. Comert. "Short-term freeway traffic parameter prediction: Application of grey system theory models," Expert Systems with Application, vol. 62, pp.284-292, Nov.2016. doi:10.1016/j.eswa.2016.06.032

[7] V. Prema, K. Uma Rao. "Development of statistical time series models for solar power prediction," Renewable Energy, vol. 83, pp.100-109, Nov.2015. doi:10.1016/j.renene.2015.03.038

[8] Y.N. Zhao, L. Ye, Z. Li, X.R. Song, Y.S. Lang, J. Su. “A novel bidirectional mechanism based on time series model for wind power forecasting," Applied Energy. vol. 177, pp. 793-803, Mar.2016. doi:10.1016/j.apenergy.2016.03.096

[9] Y.G. Zhang, P.H. Wang, P.L. Cheng, S. Lei. "Wind Speed Prediction with Wavelet Time Series Based on Lorenz Disturbance,” Advances in Electrical and Computer Engineering, vol. 17, pp.107-114, Aug. 2017. doi:10.1016/j.aece.2017.03.014

[10] F. Bre, J.M. Gimenez, V.D. Fanchinotti. "Prediction of wind pressure coefficients on building surfaces using artificial neural networks," Energy and Buildings, vol.158, pp.1429-1441, Jan. 2018 doi:10.1016/j.enbuild.2017.11.045

[11] J. P. Jeon, C. Kim, B.D. Oh, S. J. Kim, Y.S. Kim. "Prediction of persistent hemodynamic depression after carotid angioplasty and stenting artificial neural network model,” Clinical Neurology and Neurosurgery, vol. 164, pp. 127-131, Dec.2017. doi:10.1016/j.clineuro.2017.12.005

[12] P. Ramasamy, S.S. Chandel, A.K. Yadav. "Wind speed prediction in the mountainous region of India using an artificial neural network model,” Applied Energy, vol. 80, pp.338-347, Aug.2015. doi:10.1016/j.renene.2015.02.034

[13] M. Wagarachchi, A. Karunananda. "Optimization of artificial neural network architecture using neuroplasticity,” International Journal of Artificial Intelligence, vol. 15, no. 1, pp. 112-125, 2017.

[14] L.N. Liu, Y.L. Lei. “An accurate ecological footprint analysis and prediction for Beijing based on SVM model,” Ecological Informatics, vol. 17, pp.1574-9541, Jan. 2018. doi:10.1016/j.ecoinf.2018.01.003
[15] B.A. Moghram, E. Nabil, A. Badr. “Ab-initio conformational epitope structure prediction using genetic algorithm and SVM for vaccine design," Computer Methods and Programs in Biomedicine, vol. 153, pp. 161-170, Jan. 2018. doi:10.1016/j.cmpb.2017.10.011

[16] D. Martín, B. Caballero, R. Haber. "Optimal tuning of a networked linear controller using a multi-objective genetic algorithm and its application to one complex electromechanical process,” International Journal of Innovative Computing, Information and Control, vol. 5, no. 10 (B) pp. 3405-3414, 2009.

[17] R.E. Precup, S. Doboli, S. Preitl. "Stability analysis and development of a class of fuzzy control systems," Engineering Applications of Artificial Intelligence, vol. 13, no. 3, pp. 237-247, 2000.

[18] A. Karniel, G.F. Inbar, "Human motor control: learning to control a time-varying, nonlinear, many-to-one system," IEEE Transactions on Systems, Man, and Cybernetics, Part C, vol. 30, no. 1, pp. 1-11, 2000.

[19] J. Naik, P. Satapathy, P.K. Dash. "Short-term wind speed and wind power prediction using hybrid empirical mode decomposition and kernel ridge regression,” Applied Soft Computing, pp.ASOC-4606, Dec.2017. doi:10.1016/j.asoc.2017.12.010

[20] C. Zhang, H.K. Wei, J.S. Zhao, T.H. Liu, T.T. Zhu, K.J. Zhang. "Short-term wind speed forecasting using empirical mode decomposition and feature selection,” Renewable Energy, vol.96, pp.727-737, May 2016. doi:10.1016/j.renene.2016.05.023

[21] W.Y. Duan, Y. Han, L.M. Huang, B.B. Zhao, M.H. Wang. “A hybrid EMD-SVR model for the short-term prediction of significant wave height,” Ocean Engineering, vol. 124, pp. 54-73, Sep.2016. doi:10.1016/j.oceaneng.2016.05.049

[22] H. Liu, H.Q. Tian, Y.F. Li. "An EMD-recursive ARIMA method to predict wind speed for railway strong wind warning system,” Journal of Wind Engineering and Industrial Aerodynamics, vol. 141, pp. 2738, Mar.2015. doi:10.1016/.jweia.2015.02.004

[23] X.B. Kong, X.J. Liu, R.F. Shi, K. Y.Lee. "Wind speed prediction using reduced support vector machines with feature selection," Neurocomputing, vol.169, 449-456, Apr.2015. doi:10.1016/j.neucom.2014.09.090

[24] A. Baghban, M. N. Kardani, S. Habibzadeh. "Prediction viscosity of ionic liquids using a hybrid LSSVM and group contribution method," Journal of Molecular liquids, vol. 236, pp.452 -464, April 2017. doi:10.1016/j.molliq.2017.04.019

[25] R. Langone, C. Alzate, B.D. Ketelaere, Jonas Vlasselaer, Wannes Meert. "LS-SVM based spectral clustering and regression for predicting maintenance of industrial machines," Engineering Applications of Artificial Intelligence, vol.37, pp.268-278, Jan. 2015. doi:10.1016/j.engappai.2014.09.008

[26] A. Glowacz. "Recognition of Acoustic Signals of Loaded Synchronous Motor Using FFT, MSAF-5 and LSVM," Archives of Acoustics, vol.40, pp. 197-203, Feb. 2015. doi: 10.1515/aoa-20150022

[27] A. Glowacz, W. Glowacz, Z. Glowacz, J. Kozik. "Early fault diagnosis of bearing and stator faults of the single-phase induction motor using acoustic signals," Measurement, vol.113, pp.1-9, Jan.2018. DOI: 10.1016/j.measurement.2017.08.036

[28] Sotavento Galicia [DB/OL].

[2014-11-30] http://www.sotaventogalicia.com/en

[29] Y.G. Zhang, J.Y. Yang, K.C. Wang, Z.P. Wang, Y.D. Wang, "Improved wind prediction based on the Lorenz system," Renewable Energy, vol. 81, pp. 219-226, Mar. 2015. doi: 10.1016/j.renene.2015. 03.039 